The minimal curvaton-higgs model

This content has been downloaded from IOPscience. Please scroll down to see the full text. JCAP01(2014)006

(http://iopscience.iop.org/1475-7516/2014/01/006)

View the table of contents for this issue, or go to the journal homepage for more

Download details:

IP Address: 134.94.122.242

This content was downloaded on 28/07/2014 at 08:07

Please note that terms and conditions apply. 


\title{
The minimal curvaton-higgs model
}

\author{
Kari Enquist, ${ }^{a}$ Rose N. Lerner ${ }^{a, b}$ and Tomo Takahashi ${ }^{c}$ \\ ${ }^{a}$ Physics Department, University of Helsinki and Helsinki Institute of Physics, \\ P.O. Box 64, FI-00014, Helsinki, Finland \\ ${ }^{b}$ Deutsches Elektronen-Synchrotron DESY, \\ 22607 Hamburg, Germany \\ ${ }^{c}$ Department of Physics, Saga University, \\ Saga 840-8502, Japan \\ E-mail: kari.enqvist@helsinki.fi, rose.lerner@desy.de, tomot@cc.saga-u.ac.jp
}

Received October 11, 2013

Accepted December 16, 2013

Published January 7, 2014

\begin{abstract}
We present the first full study of the minimal curvaton-higgs (MCH) model, which is a minimal interpretation of the curvaton scenario with one real scalar coupled to the standard model Higgs boson. The standard model coupling allows the dynamics of the model to be determined in detail, including effects from the thermal background and from radiative corrections to the potential. The relevant mechanisms for curvaton decay are incomplete non-perturbative decay (delayed by thermal blocking), followed by decay via a dimension-5 non-renormalisable operator. To avoid spoiling the predictions of big bang nucleosynthesis, we find the "bare" curvaton mass to be $m_{\sigma} \geq 8 \times 10^{4} \mathrm{GeV}$. To match observational data from Planck there is an upper limit on the curvaton-higgs coupling $g$, between $10^{-3}$ and $10^{-2}$, depending on the mass. This is due to interactions with the thermal background. We find that typically non-Gaussianities are small but that if $f_{\mathrm{NL}}$ is observed in the near future then $m_{\sigma} \lesssim 5 \times 10^{9} \mathrm{GeV}$, depending on Hubble scale during inflation. In a thermal dark matter model, the lower bound on $m_{\sigma}$ can increase substantially. The parameter space may also be affected once the baryogenesis mechanism is specified.
\end{abstract}

Keywords: inflation, particle physics - cosmology connection, non-gaussianity, cosmology of theories beyond the SM

ArXiv ePrint: 1310.1374 


\section{Contents}

1 Introduction 1

2 MCH model to one loop $\quad 2$

2.1 Scalar potential 2

2.2 Non-perturbative and perturvative decays 3

2.2.1 Non-perturbative decay 4

2.2.2 Gravitational strength decay 5

2.2.3 Perturbative scatterings with the thermal background 6

$\begin{array}{lll}3 & \text { The curvature perturbation and non-Gaussianity } & 6\end{array}$

4 Results $\quad 9$

$\begin{array}{llr}4.1 & \text { Solution } 1 & 10\end{array}$

4.2 Solution $2 \quad 11$

5 Conclusion

\section{Introduction}

In the curvaton scenario [1-3], the primordial density perturbations are generated after a period of primordial inflation, the details of which need not be specified. ${ }^{1}$ Once inflation is over and the inflaton has decayed into radiation, which we assume to consist of the standard model degrees of freedom, the curvaton field begins to evolve. Eventually it starts to oscillate in its potential, and in the process the inflationary perturbations imprinted on the curvaton field grow. The final amplitude of the perturbation, as well as its statistical properties, is defined by the time of the curvaton decay. The decay products inherit the field perturbation, which is then converted into an adiabatic curvature perturbation by subsequent thermalisation. Recently, attempts have been made to determine the curvaton decay width in terms of model parameters, and also to connect the curvaton model with the standard model and other particle physics scenarios $[6,7]$. Here the assumption is that the curvaton provides all of the observed perturbation; for scenarios where both the inflaton and the curvaton contribute, see [8-13].

In order to obtain the observed curvature perturbation amplitude of $\zeta=4.7 \times 10^{-5}$ [14], the oscillating homogeneous curvaton condensate must be relatively long lived. Moreover, to obtain realistic estimates for the properties of the curvature perturbation, one should know both the form of the curvaton potential and the mechanism by which the curvaton decays. In addition to the amplitude of the curvature perturbation, non-Gaussianity can also give a stringent constraint on the curvaton model [15]. Recent Planck data has provided a constraint on a non-linearity parameter $f_{\mathrm{NL}}$, which represents the amplitude of the bispectrum of the curvature perturbation. This constraint is $-8.9<f_{\mathrm{NL}}<14.3$ (95\% C.L.) [16] for the local type, which excludes a large parameter space of the curvaton model with a quadratic

\footnotetext{
${ }^{1}$ The spectral index $n_{s}$ is determined by both the inflaton and the curvaton potential. Although $n_{s}$ is now severely constrained by Planck data [4], we do not calculate it because we have not specified the inflaton potential. For the discussion on the spectral index in the curvaton or a spectator field model, see [5].
} 
potential [16]. However, curvaton self-interactions may change the naive expectations for non-Gaussianity parameters considerably [17-29] and the extent of the exluded region in the parameter space in such a case remains to be studied.

Our aim in this paper is to consider carefully the predictions from a very specific implementation of the curvaton scenario, consisting of the standard model plus one curvaton field that is coupled to the Higgs boson. We will limit ourselves to the simplest quadratic curvaton model, but will account for the effective self-interaction arising through radiative corrections induced by the curvaton-higgs coupling. With such a specific model, we are able to calculate the decay width of the curvaton using particle physics. The dominant forms of decay are resonant production of higgses and decay to the standard model via dimension-5 operators. Using this information, we are able to make accurate predictions for the non-Gaussianity parameters in this model.

The structure of this paper is as follows. In the next section, we describe the $\mathrm{MCH}$ model. We give the expressions for the potential and the decay rates, which are necessary to calculate the density perturbation. Then in section 3 , we summarize the formalism to calculate the curvature perturbation and its correlation functions such as the power and bi- spectra, which will be used to test the model with observational data from Planck. In section 4 , we give a prediction for the power spectrum and non-linearity parameter $f_{\mathrm{NL}}$ and discuss the constraints on the parameters in the model. The final section is devoted to our conclusions.

\section{MCH model to one loop}

In order to produce the observed adiabatic curvature perturbation, the curvaton must at some point decay to radiation. Therefore it must be coupled to other fields. Assuming a gauge singlet curvaton, the only renormalizable way to couple it to the standard model is via the Higgs field. The simplest possibility is then given by the potential

$$
V_{0}=\frac{1}{2} m_{\sigma}^{2} \sigma^{2}+g^{2} \sigma^{2} \Phi^{\dagger} \Phi,
$$

where $g$ is a free coupling constant, and $\Phi$ is the complex Higgs doublet. Within a few thousand Hubble times after inflation, the higgs settles to the minimum of its potential [30]. Thus, for the purposes of this paper, we can consider only the physical (real) higgs field, which has a normalisation of $1 / \sqrt{2}$ compared to $\Phi$. In principle, we could also have assumed a trilinear coupling of the type $\sigma \Phi^{\dagger} \Phi$, but this could have been done only at the cost of introducing a new mass scale. Such a term can be forbidden by imposing the global symmetry $\sigma \rightarrow-\sigma$ in the scalar sector. The dimension-5 operator introduced later (eq. (2.13)) does not respect the symmetry $\sigma \rightarrow-\sigma$ of the potential (eq. (2.1)). However, it is assumed that gravity breaks all global symmetries. We have also assumed the bare quartic coupling is negligible (an effective quartic coupling is generated by loop corrections). With this setup, we now can calculate the effective potential and the decay rate for the curvaton, including quantum and thermal corrections.

\subsection{Scalar potential}

Both thermal corrections and quantum correction of the Coleman-Weinberg type exist because of the curvaton-higgs coupling in (2.1). (There are also contributions from curvatononly terms.) Thus the simplest complete curvaton potential reads

$$
V_{\text {eff }}(\sigma, T)=V_{0}(\sigma)+\Delta V(\sigma)+V_{T}(\sigma, T),
$$


where

$$
V_{T}=\frac{1}{24} g^{2} T^{2} \sigma^{2},
$$

is the (one-loop) thermal correction due to physical higgs loops, valid when the effective higgs mass $^{2} m_{H} \ll T$. The one-loop Coleman-Weinberg correction is given by

$$
\Delta V(\sigma)=\frac{\left(g^{2} \sigma^{2}+m_{h}^{2}\right)^{2}}{64 \pi^{2}} \log \left(\frac{g^{2} \sigma^{2}+m_{h}^{2}}{\mu^{2}}\right),
$$

where $m_{h}=126 \mathrm{GeV}$ is the higgs mass excluding interaction terms and $\mu$ is the renormalisation scale. For our numerical studies, we choose $\mu=m_{h}$. This is an arbitrary choice in principle, but different values cause the couplings to be defined at different scales. We do not consider the running of couplings here, because it is a higher-order effect than the existence of the Coleman-Weinberg potential.

Given the curvaton potential (2.2), one could define an effective decay rate of the curvaton, $\Gamma_{\text {eff }}$, which is a dynamical quantity that depends parametrically on the initial curvaton field value $\sigma_{*}$ and the Hubble rate at the end of inflation $H_{*}$ (which determines the inflaton reheating temperature). Moreover, the effective decay rate also depends on the parameters of the potential so that ${ }^{3} \Gamma_{\text {eff }}=\Gamma_{\text {eff }}\left(\sigma_{*}, H_{*}, m_{\sigma}, g\right)$; the actual number can be computed by solving the equations of motion. The final curvature perturbation is then $\zeta=\zeta\left(\sigma_{*}, H_{*}, m_{\sigma}, \Gamma_{\text {eff }}\right)$ with

$$
\zeta=\zeta_{G}+\frac{3}{5} f_{\mathrm{NL}} \zeta_{G}^{2}+\ldots \equiv \delta N=\frac{\partial N}{\partial \sigma}+\frac{1}{2} \frac{\partial^{2} N}{\partial \sigma^{2}}+\ldots
$$

where the amplitude of $\zeta$ is fixed by $\zeta=4.7 \times 10^{-5}$ [14]. This constraint results in a 3D surface in the space of the parameters on which the non-gaussianity parameter $f_{\mathrm{NL}}$ can be determined by numerically solving the equations of motion. In principle the curvaton contribution to other variables such as the spectral index of the primordial perturbations could be used to constrain the inflaton contibutions.

\section{$2.2 \quad$ Non-perturbative and perturvative decays}

Given the potential (2.1), the curvaton can only decay into higgses, and this can happen via two different mechanisms. The first is is by a non-perturbative resonant process whereby the oscillating curvaton field excites higgs quanta. The second is by perturbative scatterings with the thermal background of higgses formed in inflaton decay. This has been discussed for the curvaton scenario in $[6,7]$; for generic studies of the decay of a scalar field oscillating in thermal background, see e.g. [31-34]. In addition, there exists a third possibility, decay through higher order non-renormalizable operators. The simplest possibility is to postulate decay by gravitational strength interactions, which to lowest order are mediated via dimension- 5 operators. (Note that such operators are likely to break global symmetries like $\sigma \rightarrow-\sigma$.) The total effective decay rate is thus given by

$$
\Gamma_{\text {eff }}=\Gamma_{\mathrm{NP}}+\Gamma_{5}+\Gamma_{\text {pert }},
$$

where $\Gamma_{\mathrm{NP}}$ represents the contribution from non-perturbative decay, $\Gamma_{5}$ the contribution from dimension- 5 operators, and $\Gamma_{\text {pert }}$ the contribution from perturbative scattering with the thermal bath. These are all discussed in the following sections.

\footnotetext{
${ }^{2}$ In principle the dependence of the thermal mass on temperature is more complicated (see e.g. [31]).

${ }^{3}$ Although the Coleman-Weinberg correction eq. (2.4) introduces a dependence on $m_{h}$, this turns out to be negligible because eq. (2.4) turns out not to play a big role in the curvaton's evolution.
} 


\subsubsection{Non-perturbative decay}

Non-perturbative decay can occur and was discussed in detail for this model in $[6,7]$ (for earlier discussion of non-perturbative curvaton decay, see [35-38]). For this, it is essential to take into account corrections due to the thermal background of the inflaton decay products. These affect both the curvaton potential and the dispersion relation of the higgs, which determines the nature and existence of the resonance [6]. The resonance occurs for certain momentum modes and can be labeled either as a broad or a narrow resonance. The resonance parameter is defined by

$$
q(t)=\left(\frac{g \Sigma(t)}{2 m_{\sigma}}\right)^{2},
$$

where $\Sigma(t)$ is the amplitude of the curvaton condensate at time $t$. A broad resonance has $q \gg 1$ whereas a narrow resonance has $q \ll 1$. The nature and effectiveness of the resonance depends on the curvaton initial conditions, its mass, its coupling to the higgs, and the subsequent evolution. In the absence of a thermal background, the curvaton would undergo broad resonance, and the timescale of energy transfer from the curvaton to the higgs would be fast. However, thermal corrections to the higgs mass can often block the curvaton decay for a long time [6]. As a consequence, the resonance parameter evolves, and typically the resonance is narrow at the time when the resonant production of higgses becomes possible.

The onset of narrow resonance occurs when the temperature satisfies

$$
T_{\mathrm{NR}} \simeq \frac{m_{\sigma}}{g_{T}},
$$

where $g_{T}^{2} \simeq 0.1$ [39] is the effective thermal higgs coupling summed over all SM degrees of freedom. When the resonance is first unblocked, the center of the resonance band is near $k=0$ ( $k$ is comoving momenta). As the thermal blocking becomes less important, the center of the band moves towards $(k / a)=m_{\sigma}$. The width of the resonance band is always given by $2 q(t)$; this decreases with time. Thus, modes $k$ that are initially inside the resonance band will eventually leave it. Modes which are initially outside the band can enter and then leave. Because the narrow resonance is a resonant effect, the time a mode spends in the resonance band is important. Most energy is transferred to the higgs in modes which are initially outside the resonance band. The condition for efficient transfer of energy from the curvaton to the higgs is given by [7]

$$
q^{2}\left(t_{\mathrm{NR}}\right) m_{\sigma} t_{\mathrm{NR}} \gtrsim 1,
$$

where $t_{\mathrm{NR}}$ is the time when eq. (2.8) is satisfied. If eq. (2.9) is satisfied, then the resonance is efficient. Although it is possible to determine the time when the resonance becomes unblocked and the curvaton condensate starts decaying into higgses, a rigorous numerical simulation is required to determine precisely when the decay stops, or the time when backreaction becomes important. This is beyond the scope of this project. However, it is known from simulations $[40,41]$ that some fraction $F_{\mathrm{NP}}$ of the condensate (or some non-relativistic particles) remains, typically between 1 and $10 \%$. In a study of curvaton preheating it was found that about $5 \%$ of the condensate remains [35], and therefore for definiteness in this paper we choose $F_{\mathrm{NP}}=0.05$ when eq. (2.9) is satisfied and $F_{\mathrm{NP}}=1$ when eq. (2.9) is not satisfied. These assumptions have a negligible impact on the final results, because the curvature perturbation is dominated by the final decay. We have checked that our results are not sensitive to changes in $F_{\mathrm{NP}}$. 
To evaluate $\Gamma_{\mathrm{NR}}$, we need to numerically follow the evolution of both the curvaton field and the background, because $q(t)$ depends on the amplitude of the curvaton condensate. However, we can check the numerical results by making an analytic evaluation of $q\left(t_{\mathrm{NR}}\right)$ in three separate cases. Applying the scaling law for energy densities of the curvaton and radiation, we find

$$
q\left(t_{\mathrm{NR}}\right) \sim\left(\frac{g}{g_{T}}\right)^{3}\left(\frac{\sigma_{*}}{M_{\mathrm{pl}}}\right)^{2}\left(\frac{M_{\mathrm{pl}}}{H_{*}}\right),
$$

for the case where oscillations begin immediately after inflation in the thermal potential eq. (2.3). In the second case there is a period of slow roll before oscillations begin in the thermal potential eq. (2.3), and we find

$$
q\left(t_{\mathrm{NR}}\right) \sim \frac{g}{g_{T}^{3}}\left(\frac{\sigma_{*}}{M_{\mathrm{pl}}}\right)^{2} .
$$

Finally, in the case where there is slow roll followed by oscillations in the quadratic potential eq. (2.1), we find

$$
q\left(t_{\mathrm{NR}}\right) \sim \frac{g^{2}}{g_{T}^{3}}\left(\frac{\sigma_{*}}{M_{\mathrm{pl}}}\right)^{2}\left(\frac{M_{\mathrm{pl}}}{m_{\sigma}}\right)^{1 / 2} .
$$

The numerical and analytical results are in good agreement.

\subsubsection{Gravitational strength decay}

In addition to the renormalizable coupling to the higgs, higher order effective operators are expected to exist since there is no symmetry that would explicitly forbid them. These include the curvaton coupling to standard model fermions $f$ through dimension- 5 operators such as

$$
\mathcal{L}_{5} \propto \frac{1}{M_{P}} \sigma \bar{f} \Phi f .
$$

This is just one example; there would also be $d=5$ operators involving gauge fields. On dimensional grounds and neglecting possible coefficients, the $d=5$ operators in combination yield a curvaton decay rate of

$$
\Gamma_{5} \approx \frac{m_{\sigma}^{3}}{M_{P}^{2}} .
$$

Note that we have chosen $M_{P}$ as the scale of the higher order operators, and therefore no new parameters have been introduced. In principle, the scale is unknown. ${ }^{4}$ Since we implicitly assume that, apart from the curvaton, there is no new physics beyond the standard model and Einstein gravity, choosing $M_{P}$ as the scale of non-renormalizable physics seems justified.

Although the decay rate (2.14) is Planck suppressed, it can nevertheless be more important than the non-perturbative decay. Moreover, after backreaction shuts down the resonant production of higgses, in the minimal scenario presented here it is the higher-order operators that will be responsible for completing the curvaton decay.

If dimension-5 operators were forbidden for some reason, the effective decay rate by dimension- 6 operators would be (again on dimensional grounds and neglecting coefficients)

$$
\Gamma_{6} \approx \frac{m_{\sigma}^{5}}{M_{P}^{4}} .
$$

\footnotetext{
${ }^{4}$ However, it should be much larger than the curvaton mass, which should be smaller than the Hubble rate during inflation, which could be as high as $10^{12} \mathrm{GeV}$.
} 
We require the curvaton to decay before BBN to avoid spoiling the predictions [42]. Specifically, it should decay before the neutrinos decouple at $T=4 \mathrm{MeV}$ [43]. These limits are found by assuming $H(t) \propto T^{2}$ and calculating when $H(t)=\Gamma_{5}$ or $\Gamma_{6}$. For the dimension5 operator, this corresponds to requiring $m_{\sigma} \gtrsim 8 \times 10^{4} \mathrm{GeV}$. However, for the dimension- 6 operator, the condition on $m_{\sigma}$ is much stronger, giving $m_{\sigma} \gtrsim 4 \times 10^{10} \mathrm{GeV}$. This would only allow a small window of curvaton masses; the upper bound is given by $m_{\sigma}<H_{*}$. However, we presume that the dimension-5 operators are not forbidden, and thus a substantial amount of parameter space remains.

\subsubsection{Perturbative scatterings with the thermal background}

The presence of a thermal bath means that decay processes for the curvaton include perturbative scatterings with the thermal background, the importance of which were first pointed out in [44]. We calculate the decay width for this, also including $1 \rightarrow 3$ particle decays and production of $k=0$ modes from the thermal background. One finds [45, 46]

$$
\Gamma_{\text {pert }}=\frac{1}{576 \pi} \frac{g^{4} T^{2}}{m_{\sigma}(T)} .
$$

We now consider the two cases for initial effective mass at the temperature $T_{*}$, where $T_{*}$ is the reheating temperature assuming instant reheating. If $m_{\sigma}\left(T_{*}\right)=m_{\sigma}$, then because $H(t) \propto T^{2} / M_{\mathrm{pl}}$, decay by these perturbative interactions either never occurs, or occurs immediately after the thermal background has been produced. If curvaton decay occurs immediately, then the curvaton model is ruled out because the relative fraction of the curvaton energy density has not had time to increase to satisfy the normalization $\zeta=4.7 \times 10^{-5}$. This rules out large $g$, and depends on $m_{\sigma}$. If instead $m_{\sigma}\left(T_{*}\right)=g T / \sqrt{12}$, then we should determine whether these perturbative interactions can occur before $T \simeq m_{\sigma} / g$, which marks the transition to $m(T)=m_{\sigma}$. In that case, the curvaton decays while both $\rho_{\sigma}$ and $\rho_{\text {rad }}$ scale $\propto a^{-4}$. This means that the relative fraction of the curvaton energy density cannot grow, and that the curvaton model is ruled out in this region. The condition for this to occur is given by

$$
g \geq 4.9 g_{*}^{1 / 8}\left(\frac{m_{\sigma}}{M_{\mathrm{pl}}}\right)^{1 / 4},
$$

where $g_{*}=107.75$ is the effective number of degrees of freedom.

\section{The curvature perturbation and non-Gaussianity}

Now we discuss the curvature perturbation in the model. We adopt the $\delta N$ formalism [47-50] to calculate the curvature perturbation up to the 3rd order:

$$
\zeta=N_{\sigma} \delta \sigma_{*}+\frac{1}{2} N_{\sigma \sigma}\left(\delta \sigma_{*}\right)^{2}+\frac{1}{6} N_{\sigma \sigma \sigma}\left(\delta \sigma_{*}\right)^{3},
$$

where $N_{\sigma}=d N / d \sigma_{*}$ and so on. Then the power, bi- and tri-spectra are defined respectively as

$$
\begin{aligned}
\left\langle\zeta\left(\boldsymbol{k}_{1}\right) \zeta\left(\boldsymbol{k}_{2}\right)\right\rangle & =(2 \pi)^{2} P_{\zeta}\left(k_{1}\right) \delta\left(\boldsymbol{k}_{1}+\boldsymbol{k}_{2}\right), \\
\left\langle\zeta\left(\boldsymbol{k}_{1}\right) \zeta\left(\boldsymbol{k}_{2}\right) \zeta\left(\boldsymbol{k}_{3}\right)\right\rangle & =(2 \pi)^{2} B_{\zeta}\left(k_{1}, k_{2}, k_{3}\right) \delta\left(\boldsymbol{k}_{1}+\boldsymbol{k}_{2}+\boldsymbol{k}_{3}\right), \\
\left\langle\zeta\left(\boldsymbol{k}_{1}\right) \zeta\left(\boldsymbol{k}_{2}\right) \zeta\left(\boldsymbol{k}_{3}\right) \zeta\left(\boldsymbol{k}_{4}\right)\right\rangle & =(2 \pi)^{2} T_{\zeta}\left(k_{1}, k_{2}, k_{3}, k_{4}\right) \delta\left(\boldsymbol{k}_{1}+\boldsymbol{k}_{2}+\boldsymbol{k}_{3}+\boldsymbol{k}_{4}\right),
\end{aligned}
$$


where $B_{\zeta}$ and $T_{\zeta}$ are given by

$$
\begin{aligned}
B_{\zeta}\left(k_{1}, k_{2}, k_{3}\right)= & \frac{6}{5} f_{\mathrm{NL}}\left(P_{\zeta}\left(k_{1}\right) P_{\zeta}\left(k_{2}\right)+P_{\zeta}\left(k_{2}\right) P_{\zeta}\left(k_{3}\right)+P_{\zeta}\left(k_{3}\right) P_{\zeta}\left(k_{1}\right)\right), \\
T_{\zeta}\left(k_{1}, k_{2}, k_{3}, k_{4}\right)= & \tau_{\mathrm{NL}}\left(P_{\zeta}\left(k_{13}\right) P_{\zeta}\left(k_{3}\right) P_{\zeta}\left(k_{4}\right)+11 \text { perms. }\right) \\
& +\frac{54}{25} g_{\mathrm{NL}}\left(P_{\zeta}\left(k_{2}\right) P_{\zeta}\left(k_{3}\right) P_{\zeta}\left(k_{4}\right)+3 \text { perms. }\right),
\end{aligned}
$$

where $k_{13}=\left|\vec{k}_{1}+\vec{k}_{3}\right|$. When the curvature perturbations are generated from the curvaton field alone, the power spectrum is given by

$$
P_{\zeta}(k)=N_{\sigma}^{2} P_{\delta \sigma}(k),
$$

where $P_{\delta \sigma}$ is the power spectrum for fluctuations of the curvaton field $\delta \sigma$, which is given by

$$
P_{\delta \sigma}(k)=\frac{2 \pi^{2}}{k^{3}} \mathcal{P}_{\delta \sigma}(k) .
$$

Here $\mathcal{P}_{\delta \sigma}=(H / 2 \pi)^{2}$ with $H$ the Hubble parameter at the time of horizon crossing for a given mode $k$.

The non-linearity parameters $f_{\mathrm{NL}}$ and $g_{\mathrm{NL}}$ are given by

$$
\begin{aligned}
\frac{5}{6} f_{\mathrm{NL}} & =\frac{N_{\sigma \sigma}}{N_{\sigma}^{2}}, \\
\frac{54}{25} g_{\mathrm{NL}} & =\frac{N_{\sigma \sigma \sigma}}{N_{\sigma}^{3}}
\end{aligned}
$$

whereas $\tau_{\mathrm{NL}}=\left(5 f_{\mathrm{NL}} / 6\right)^{2}$.

For the curvaton, $\zeta$ can be expressed as [51]

$$
\begin{aligned}
\zeta_{\mathrm{cur}}= & \frac{2}{3} r_{\mathrm{dec}} \frac{\sigma_{\mathrm{osc}}^{\prime}}{\sigma_{\mathrm{osc}}} \sigma_{*}+\frac{1}{9}\left[3 r_{\mathrm{dec}}\left(1+\frac{\sigma_{\mathrm{osc}} \sigma_{\mathrm{osc}}^{\prime \prime}}{\sigma_{\mathrm{osc}}^{\prime 2}}\right)-4 r_{\mathrm{dec}}^{2}-2 r_{\mathrm{dec}}^{3}\right]\left(\frac{\sigma_{\mathrm{osc}}^{\prime}}{\sigma_{\mathrm{osc}}}\right)^{2}\left(\delta \sigma_{*}\right)^{2} \\
& +\frac{4}{81}\left[\frac{9 r_{\mathrm{dec}}}{4}\left(\frac{\sigma_{\mathrm{osc}}^{2} \sigma_{\mathrm{osc}}^{\prime \prime \prime}}{\sigma_{\mathrm{osc}}^{\prime 3}}+3 \frac{\sigma_{\mathrm{osc}} \sigma_{\mathrm{osc}}^{\prime \prime}}{\sigma_{\mathrm{osc}}^{\prime 2}}\right)-9 r_{\mathrm{dec}}^{2}\left(1+\frac{\sigma_{\mathrm{osc}} \sigma_{\mathrm{osc}}^{\prime \prime}}{\sigma_{\mathrm{osc}}^{\prime 2}}\right)\right. \\
& \left.+\frac{r_{\mathrm{dec}}^{3}}{2}\left(1-9 \frac{\sigma_{\mathrm{osc}} \sigma_{\mathrm{osc}}^{\prime \prime}}{\sigma_{\mathrm{osc}}^{\prime 2}}\right)+10 r_{\mathrm{dec}}^{4}+3 r_{\mathrm{dec}}^{5}\right]\left(\frac{\sigma_{\mathrm{osc}}^{\prime}}{\sigma_{\mathrm{osc}}}\right)^{3}\left(\delta \sigma_{*}\right)^{3}
\end{aligned}
$$

where $\sigma_{\mathrm{osc}}^{\prime}=d \sigma_{\mathrm{osc}} / d \sigma_{*}$, and $\sigma_{\mathrm{ocs}}$ indicates the field value of $\sigma$ at the time when the curvaton begins its oscillation under the bare mass term in the potential (2.2). The parameter $r(t)$ is defined by

$$
r(t) \equiv \frac{3 \rho_{\sigma}(t)}{4 \rho_{\mathrm{rad}}(t)+3 \rho_{\sigma}(t)},
$$

which roughly corresponds to the ratio of the curvaton energy density to the total. In particular, we define $r_{\mathrm{dec}} \equiv r\left(t=t_{\mathrm{dec}}\right)$, where $t_{\mathrm{dec}}$ is the time of curvaton decay. Notice that we assume that the narrow resonance converts the energy of the curvaton into radiation only partially. Thus, the curvaton totally decays into radiation only after the perturbative decay.

For the analysis presented in this paper, we follow the the number of $e$-folds numerically from just after inflation. We assume that the inflaton decays into radiation just after inflation 


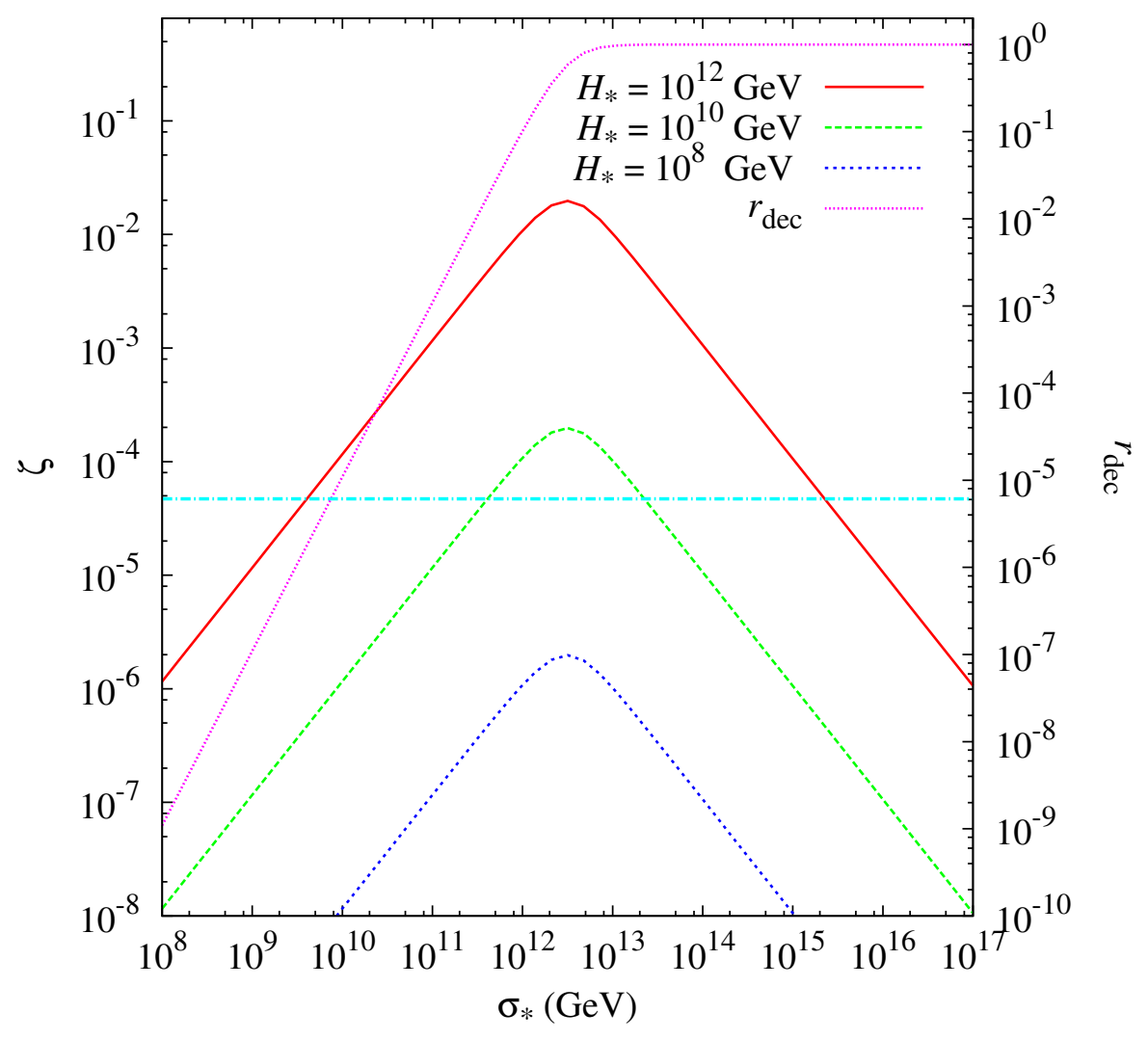

Figure 1. The dependence of $\zeta$ on the inflation scale $H_{*}$. Here we assume that $m_{\sigma}=10^{6} \mathrm{GeV}$ and $g=10^{-13}$. The horizontal line marks the observed $\zeta=4.7 \times 10^{-5}$. Also shown is the dependence of $r_{\text {dec }}$ on the initial curvaton value $\sigma_{*}$.

ends, and thus we follow the energy density of radiation and the curvaton field from the end of inflation. The number of $e$-folds $N$ can be obtained by solving the following set of equations:

$$
\begin{aligned}
\frac{d \rho_{r}}{d t}+4 H \rho_{r} & =\Gamma \rho_{\sigma}, \\
\frac{d^{2} \sigma}{d t^{2}}+(3 H+\Gamma) \frac{d \sigma}{d t}+\frac{d V}{d \sigma} & =0 \\
H^{2} & =\left(\frac{1}{a} \frac{d a}{d t}\right)^{2}=\frac{1}{3 M_{P}^{2}}\left(\rho_{r}+\rho_{\sigma}\right),
\end{aligned}
$$

where the curvaton decay rate $\Gamma$ is given by eq. (2.6). Note that $\Gamma_{\mathrm{NP}}$ is zero before the narrow resonance is unblocked, which occurs at $t=t_{\mathrm{NR}}$ (see eq. (2.8)). The initial velocity of $\sigma$ is given by the slow-roll solution.

We follow the above set of equations numerically until the time when $H=\Gamma_{5}$ is satisfied, when we can evaluate $N$ and its derivatives. Then we can calculate the power spectrum and the non-linearity parameters, which are presented in the following section.

To obtain $\zeta=4.7 \times 10^{-5}$ requires specific values of $\sigma_{*}$. There are two possible values of $\sigma_{*}$ which satisfy this requirement, as shown in figure 1 . We denote them as Solution 1 and Solution 2. Solution 1 always has $r_{\mathrm{dec}} \simeq 1$ and corresponds to what in the literature is 


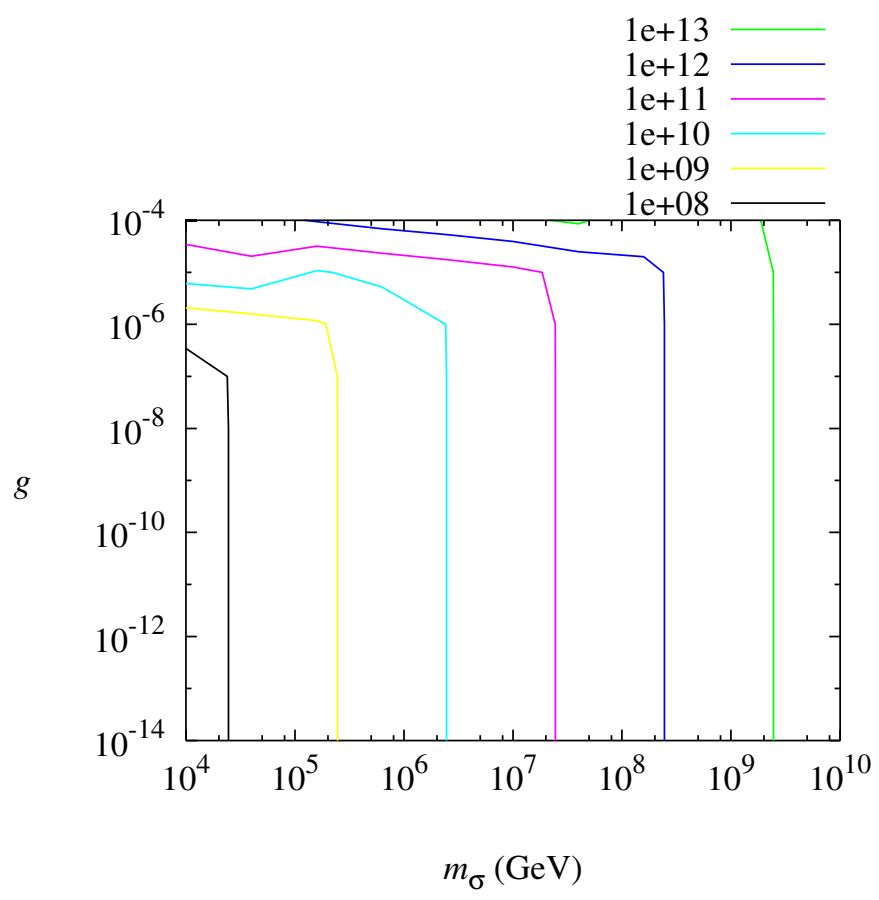

Figure 2. Contours of $\sigma_{*}$ in the case of Solution 2 that give the correct amplitude $\zeta=4.7 \times 10^{-5}$, with small $m_{\sigma}$ corresponding to small $\sigma_{*}$. Here we assume that $H_{*}=10^{12} \mathrm{GeV}$.

called the dominant curvaton. Because the non-Gaussianity parameter $f_{\mathrm{NL}} \propto 1 / r_{\mathrm{dec}}$, there is therefore no constraint from the recent Planck data (the two-sigma upper limit is $f_{\mathrm{NL}} \leq 14.3$ ).

Solution 2, which in figure 1 corresponds to the smaller value of $\sigma_{*}$, often leads to a subdominant curvaton, but for a range in $H_{*}$ can also give rise to a slightly dominant curvaton. This can be seen in figure 1 , where $r_{\text {dec }}$ associated with Solution 2 is seen to approach 1 as $H_{*}$ decreases. In this case the Planck limit on $f_{\mathrm{NL}}$ can be expected to constrain the parameter space. Note however that the parameter space of Solution 2 resides inside the parameter space of Solution 1. For illustration, in figure 2 we show the contours of $\sigma_{*}$ for Solution 2 that lead to the observed curvature perturbation. The figure is for fixed $H_{*}$; the contours look different for different Hubble rates. For Solution $1, \sigma_{*} \simeq 10^{15} \mathrm{GeV}$. This is slightly modified when the thermal correction initially dominates the potential, for large $g$ and small $m_{\sigma}$. The Colemann-Weinberg potential never dominates the potential, and we find that it also has a negligible affect on the non-Guassianity parameters. We have also checked that the Coleman-Weinberg potential does not make the curvaton heavy during inflation unless $g^{2} \gtrsim 24 \pi^{2} \zeta$ or $g \gtrsim 0.5$, which is outside the region of interest.

\section{Results}

We present results first for Solution 1, where the curvaton is always dominant at the final decay, and discuss separately Solution 2 . We fix the value of $\sigma_{*}$ such that the observed value of $\zeta$ is produced. Various constraints apply to the parameter space, including requiring decay before BBN. 


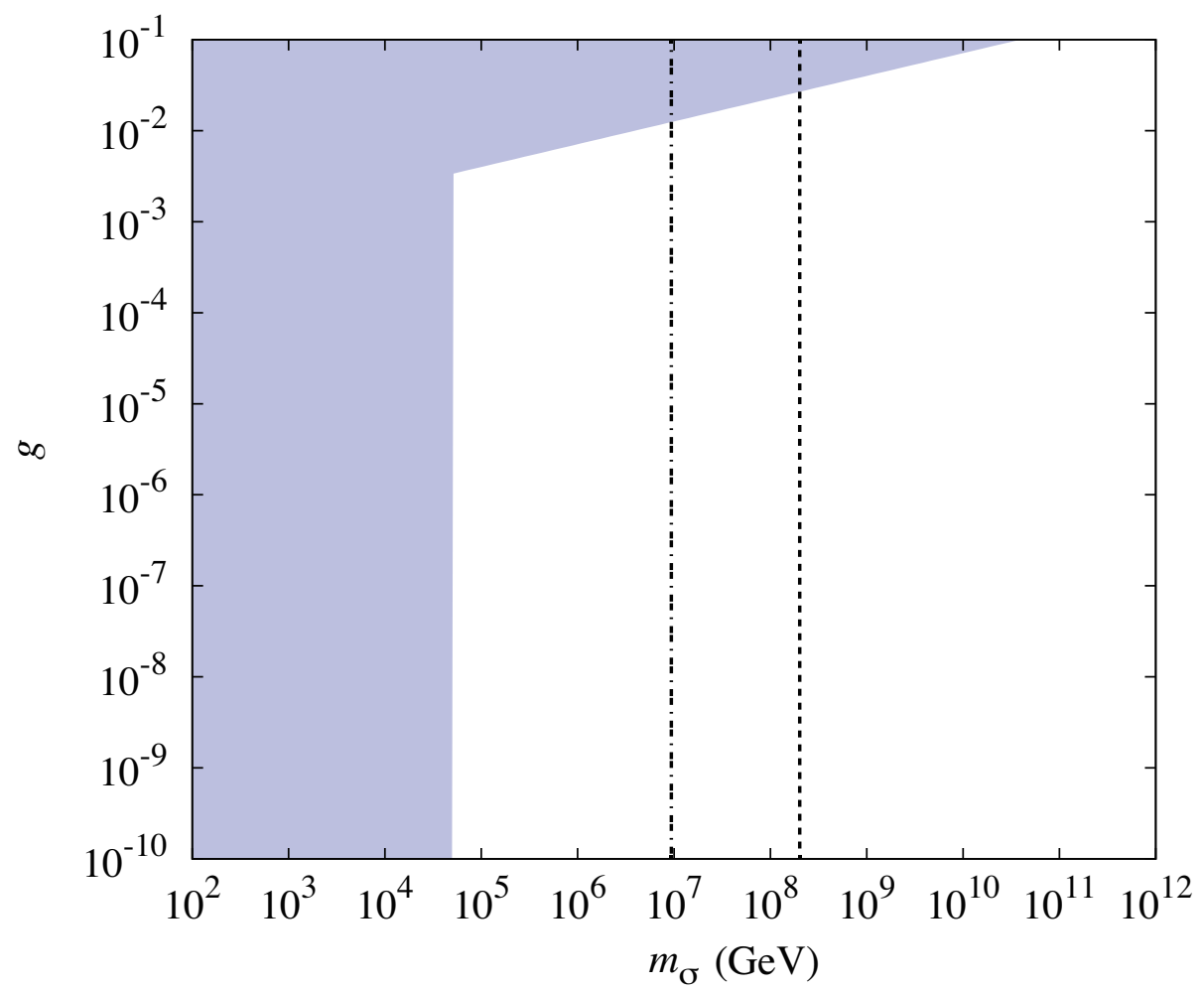

Figure 3. Parameter space for Solution 1, the dominant curvaton (white is allowed). Dashed lines show how the lower limits on $m_{\sigma}$ would increase if WIMP dark matter froze out at $10 \mathrm{GeV}$ (lower) or $1 \mathrm{TeV}$ (upper). Note that the upper bound $m_{\sigma}<H_{*}$ is not shown because it depends on $H_{*}$; also not shown for this reason is the effect of the Coleman-Weinberg potential.

We find that if the condition eq. (2.17) is satisfied, then the curvaton decays immediately via interactions with the thermal bath and the model does not work. However, these interactions with the thermal bath are not important in the rest of the parameter space. If eq. (2.17) is not satisfied, then for large $m_{\sigma}$, the dimension- 5 process occurs before the narrow resonance is unblocked. If $m_{\sigma}$ is smaller, then the narrow resonance can occur first. This could either be efficient (transferring $95 \%$ of the energy out of the curvaton) or inefficient. However, in both cases the final curvaton decay is due to the dimension-5 operator. If the curvaton is dominant at its decay, then the predictions are not particularly affected by whether there was first a narrow resonance. If the curvaton is either subdominant or only slightly dominant at decay, then the narrow resonance can affect the non-Gaussianity.

\subsection{Solution 1}

For Solution 1 the curvaton is always dominant at decay and non-Gaussianity is small. The constraints on the model are shown in figure 3, where the allowed area is white. There is also an upper limit on $m_{\sigma}$ that comes from requiring the curvaton to be light during inflation, i.e. $m_{\sigma}<H_{*}$. The upper limit on $g$ comes from requiring the interactions with the thermal bath not to immediately destroy the curvaton condensate. The lower limit on $m_{\sigma}$ comes because the final decay width is determined solely by the curvaton mass. To avoid spoiling $\mathrm{BBN}$ the curvaton must decay sufficiently early, before $T \simeq 4 \mathrm{MeV}$ (this is when $\nu_{\mu}$ and $\nu_{\tau}$ decouple [43]). There is thus a lower limit on the curvaton mass. If dark matter is a thermal 


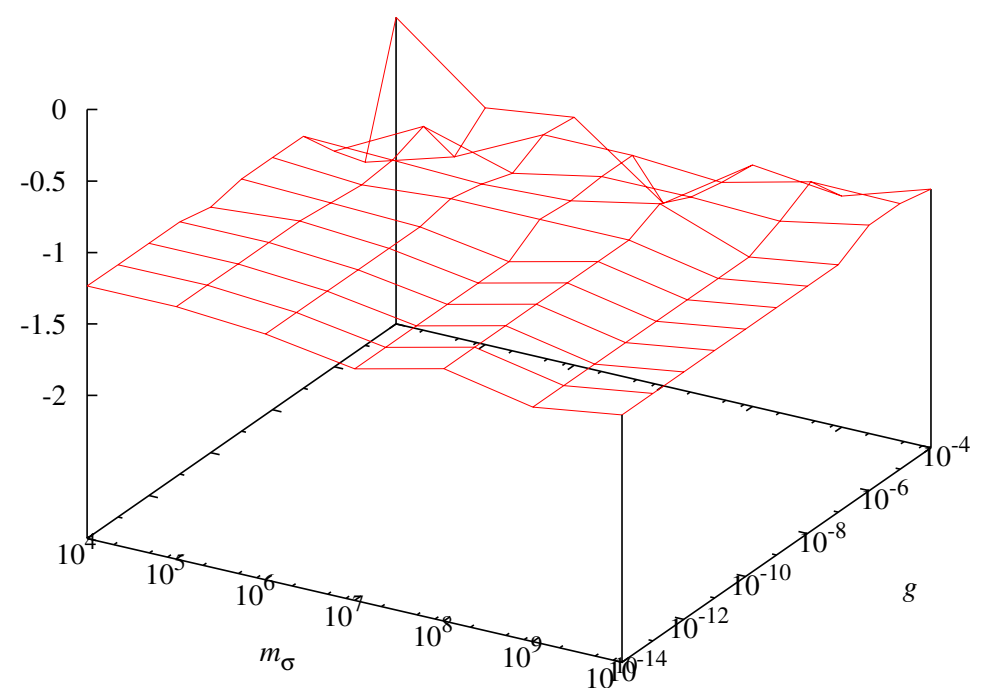

Figure 4. $f_{\mathrm{NL}}$ for Solution 1 , shown for $H_{*}=10^{12} \mathrm{GeV}$.

relic, then large isocurvature would be predicted if the curvaton decays after the dark matter freezes out. This is in contrast with observations. Thus, the dot-dashed line in figure 3 shows the lower limit on $m_{\sigma}$ for a WIMP dark matter model that freezes out at $10 \mathrm{GeV}$ while the dashed line is for a WIMP with freeze-out at $1 \mathrm{TeV}$. However, other dark matter models do not impose such a bound. A determination of the properties of dark matter will allow this constraint to be properly calculated. There could also be a lower bound from requiring the curvaton not to spoil baryogenesis. However, this is also model dependent.

The Coleman-Weinberg potential is initially dominant for Solution 1 if $g \gtrsim 0.1 \sqrt{m_{\sigma} / H_{*}}$. Figure 3 is plotted analytically and does not include this effect of the Coleman-Weinberg potential, because it depends on $H_{*}$. However, we expect that including it only changes the initial field value necessary to match observations, $\sigma_{*}$. The remaining figures show numerical results including all terms in the potential.

Although neither the Coleman-Weinberg potential nor the thermal correction dominate in most of the parameter space, they could still introduce non-Gaussianity. We have checked this carefully and found that the variations are small but possibly interesting. The precise predictions for $f_{\mathrm{NL}}$ for Solution 1 are shown in figure 4 for the case of $H_{*}=10^{12} \mathrm{GeV}$. We find a small, negative $f_{\mathrm{NL}}$ with values in the range $-1.5 \lesssim f_{\mathrm{NL}} \lesssim 0$. We have also checked that for Solution $1, g_{\mathrm{NL}}$ is unobservable with $g_{\mathrm{NL}} \lesssim 100$. We note that in all cases, both the curvature perturbation and the non-Gaussianity are produced at the second (final) decay of the curvaton. Although large non-Gaussianity can occur after the first decay, this is then diluted by the subsequent expansion. In all cases where the curvaton is dominant at decay, the first decay only has a negligible impact on the parameters. This is under the assumption that the resonance can never destroy all of the condensate. This has not been explicitly shown with a lattice simulation for our model, but is based on similar models in the literature.

\subsection{Solution 2}

When the curvaton is subdominant at both decays, large non-Gaussianity can be produced and the parameter space can be restricted by the Planck limit $f_{\mathrm{NL}} \leq 14.3$. We then find that 

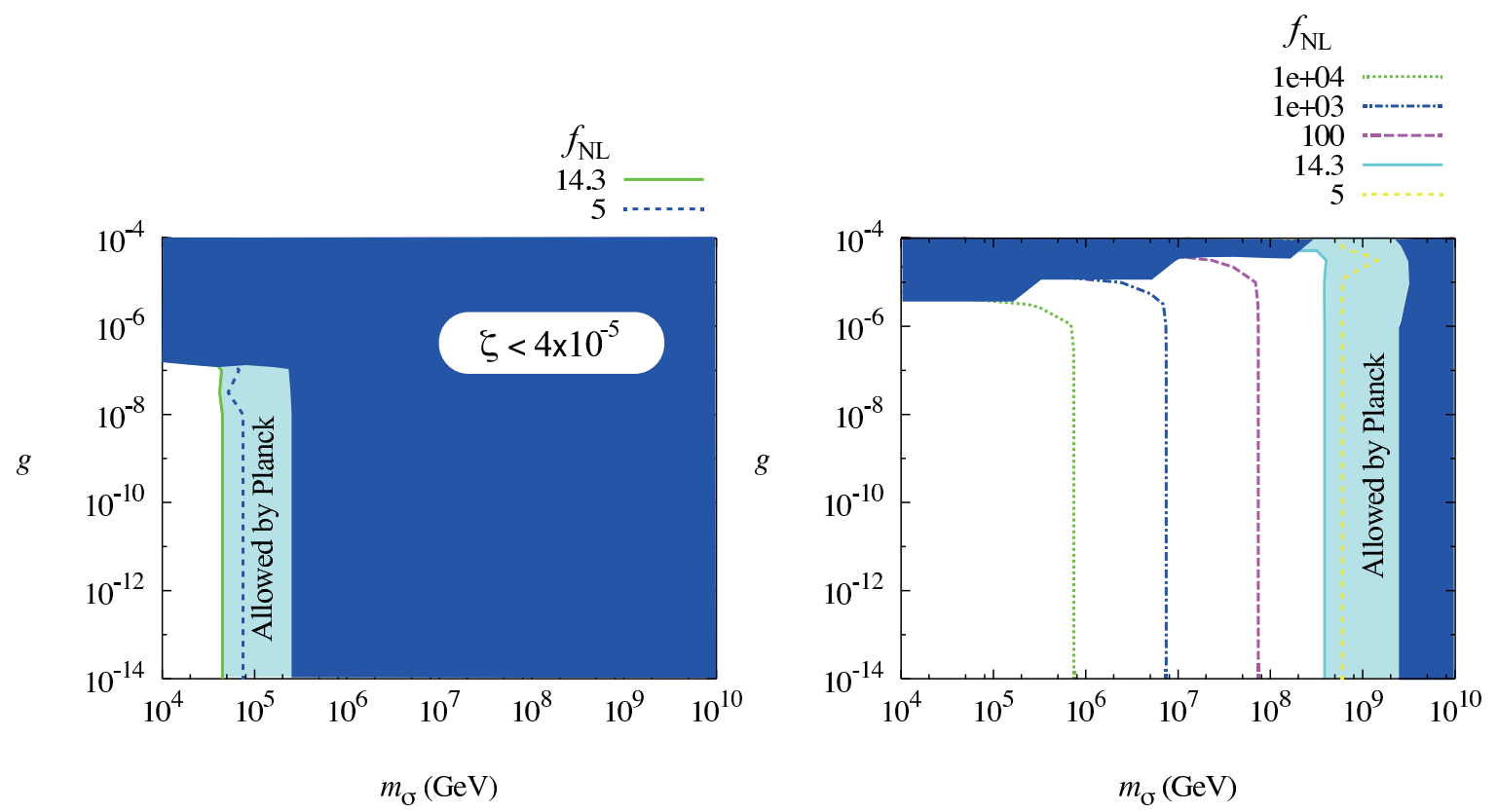

Figure 5. The allowed parameter space (pale blue / light grey) for Solution 2, with contours of $f_{\mathrm{NL}}$ shown. Left: $H_{*}=10^{9} \mathrm{GeV}$; right: $H_{*}=10^{11} \mathrm{GeV}$.

Solution 2 exists in the range $10^{9} \mathrm{GeV} \lesssim H_{*} \lesssim 10^{11} \mathrm{GeV}$. However, most of the parameter space of Solution 2 has $f_{\mathrm{NL}}$ that is not observable, e.g. $f_{\mathrm{NL}} \lesssim 5$. This is evident from figure 5 , which shows contours of $f_{\mathrm{NL}}$ for Solution 2 with $\sigma_{*}$ fixed to give the correct amplitude of $\zeta$, together with the bounds described above on $g$ and $m_{\sigma}$. The allowed space of parameters for Solution 2 is contained within the parameter space of Solution 1 depicted in figure 3. If $f_{\mathrm{NL}}$ were to be observed, it would imply a curvaton mass in the range $8 \times 10^{4} \mathrm{GeV} \lesssim m_{\sigma} \lesssim$ $5 \times 10^{9} \mathrm{GeV}$, as can be seen from figure 5 .

\section{Conclusion}

We have presented the minimal curvaton-higgs $(\mathrm{MCH})$ model and attempted to include all interactions of the model, including dimension-5 gravitationally suppressed operators. At tree-level, the curvaton is stable. However the decay mechanisms that do exist are (i) interactions with the thermal background that comes from the earlier inflaton decay, (ii) non-perturbative decay via narrow resonance, which is never completely efficient, and (iii) decay via the dimension-5 operator. Although in principle all three decay mechanisms could contribute to the observed curvature perturbation, we find that in the allowed parameter space only the dimension-5 operators impact the predictions of the model.

We demonstrated that the MCH model is viable and consistent with various constraints, and that typically the predicted non-Gaussianities are small. However, the possibility of observable $f_{\mathrm{NL}}$ still exists. More specifically, we found that the implications of the MCH model are: (a) the curvaton mass is greater than $8 \times 10^{4} \mathrm{GeV}$, (b) the curvaton-higgs coupling is constrained, depending on the mass, but $g<10^{-3}$ is always allowed, (c) if $f_{\mathrm{NL}}$ were to be observed, then $m_{\sigma} \lesssim 5 \times 10^{9} \mathrm{GeV}$, (d) if dark matter is observed and the freeze-out temperature is $10 \mathrm{GeV}(1 \mathrm{TeV})$, then the curvaton mass should be greater than $10^{7} \mathrm{GeV}$ 
$\left(10^{8} \mathrm{GeV}\right)$. If $H_{*}$ is determined to be low, and dark matter is found to be thermal relic, then the parameter space will become strongly constrained.

The MCH implementation of the curvaton scenario is important because it presents a robust model for the origin of the primordial perturbation where the particle content is fully specified. The dynamics of the model (such as the effective decay width) are not free parameters, but are given by the parameters in the Lagrangian. The model is testable with current and upcoming data and could also have implications for LHC physics. One interesting possibility would be a connection with the so-called Higgs-portal models of dark matter, where the standard model higgs is coupled to a real singlet scalar [52].

\section{Acknowledgments}

We thank Sami Nurmi and Dmitry Podolsky for comments on the fraction of the condensate remaining in preheating. TT would like to thank the Helsinki Institute of Physics for the hospitality during a visit, where part of this work was completed. The work of TT is partially supported by the Grant-in-Aid for Scientific research from the Ministry of Education, Science, Sports, and Culture, Japan, No. 23740195. KE is supported by the Academy of Finland grant 1218322; RL was supported by the Academy of Finland grant 1263714.

\section{References}

[1] K. Enqvist and M.S. Sloth, Adiabatic CMB perturbations in pre - big bang string cosmology, Nucl. Phys. B 626 (2002) 395 [hep-ph/0109214] [INSPIRE].

[2] D.H. Lyth and D. Wands, Generating the curvature perturbation without an inflaton, Phys. Lett. B 524 (2002) 5 [hep-ph/0110002] [INSPIRE].

[3] T. Moroi and T. Takahashi, Effects of cosmological moduli fields on cosmic microwave background, Phys. Lett. B 522 (2001) 215 [Erratum ibid. B 539 (2002) 303] [hep-ph/0110096] [INSPIRE].

[4] Planck collaboration, P. Ade et al., Planck 2013 results. XXII. Constraints on inflation, arXiv: 1303.5082 [INSPIRE].

[5] T. Kobayashi, F. Takahashi, T. Takahashi and M. Yamaguchi, Spectator field models in light of spectral index after Planck, JCAP 10 (2013) 042 [arXiv:1303.6255] [INSPIRE].

[6] K. Enqvist, D.G. Figueroa and R.N. Lerner, Curvaton Decay by Resonant Production of the Standard Model Higgs, JCAP 01 (2013) 040 [arXiv:1211.5028] [INSPIRE].

[7] K. Enqvist, R.N. Lerner and S. Rusak, Reheating dynamics affects non-perturbative decay of spectator fields, JCAP 11 (2013) 034 [arXiv: 1308.3321] [INSPIRE].

[8] D. Langlois and F. Vernizzi, Mixed inflaton and curvaton perturbations, Phys. Rev. D 70 (2004) 063522 [astro-ph/0403258] [INSPIRE].

[9] T. Moroi, T. Takahashi and Y. Toyoda, Relaxing constraints on inflation models with curvaton, Phys. Rev. D 72 (2005) 023502 [hep-ph/0501007] [INSPIRE].

[10] T. Moroi and T. Takahashi, Implications of the curvaton on inflationary cosmology, Phys. Rev. D 72 (2005) 023505 [astro-ph/0505339] [INSPIRE].

[11] K. Ichikawa, T. Suyama, T. Takahashi and M. Yamaguchi, Non-Gaussianity, Spectral Index and Tensor Modes in Mixed Inflaton and Curvaton Models, Phys. Rev. D 78 (2008) 023513 [arXiv: 0802.4138] [INSPIRE]. 
[12] T. Suyama, T. Takahashi, M. Yamaguchi and S. Yokoyama, On Classification of Models of Large Local-Type Non-Gaussianity, JCAP 12 (2010) 030 [arXiv:1009.1979] [INSPIRE].

[13] K. Enqvist and T. Takahashi, Mixed Inflaton and Spectator Field Models after Planck, JCAP 10 (2013) 034 [arXiv: 1306.5958] [INSPIRE].

[14] Planck collaboration, P. Ade et al., Planck 2013 results. XVI. Cosmological parameters, arXiv:1303.5076 [INSPIRE].

[15] D.H. Lyth and Y. Rodriguez, Non-Gaussianity from the second-order cosmological perturbation, Phys. Rev. D 71 (2005) 123508 [astro-ph/0502578] [INSPIRE].

[16] Planck collaboration, P. Ade et al., Planck 2013 Results. XXIV. Constraints on primordial non-Gaussianity, arXiv:1303.5084 [INSPIRE].

[17] K. Enqvist and S. Nurmi, Non-Gaussianity in curvaton models with nearly quadratic potential, JCAP 10 (2005) 013 [astro-ph/0508573] [INSPIRE].

[18] K. Enqvist and T. Takahashi, Signatures of Non-Gaussianity in the Curvaton Model, JCAP 09 (2008) 012 [arXiv:0807.3069] [INSPIRE].

[19] Q.-G. Huang, Curvaton with Polynomial Potential, JCAP 11 (2008) 005 [arXiv:0808.1793] [INSPIRE].

[20] M. Kawasaki, K. Nakayama and F. Takahashi, Hilltop Non-Gaussianity, JCAP 01 (2009) 026 [arXiv:0810.1585] [INSPIRE].

[21] P. Chingangbam and Q.-G. Huang, The Curvature Perturbation in the Axion-type Curvaton Model, JCAP 04 (2009) 031 [arXiv:0902.2619] [INSPIRE].

[22] K. Enqvist and T. Takahashi, Effect of Background Evolution on the Curvaton Non-Gaussianity, JCAP 12 (2009) 001 [arXiv:0909.5362] [INSPIRE].

[23] K. Enqvist, A. Mazumdar and O. Taanila, The TeV-mass curvaton, JCAP 09 (2010) 030 [arXiv: 1007.0657] [INSPIRE].

[24] K. Enqvist, S. Nurmi, O. Taanila and T. Takahashi, Non-Gaussian Fingerprints of Self-Interacting Curvaton, JCAP 04 (2010) 009 [arXiv:0912.4657] [INSPIRE].

[25] J. Fonseca and D. Wands, Non-Gaussianity and Gravitational Waves from Quadratic and Self-interacting Curvaton, Phys. Rev. D 83 (2011) 064025 [arXiv:1101.1254] [InSPIRE].

[26] C.T. Byrnes, K. Enqvist, S. Nurmi and T. Takahashi, Strongly scale-dependent polyspectra from curvaton self-interactions, JCAP 11 (2011) 011 [arXiv:1108.2708] [INSPIRE].

[27] M. Kawasaki, T. Kobayashi and F. Takahashi, Non-Gaussianity from Curvatons Revisited, Phys. Rev. D 84 (2011) 123506 [arXiv:1107.6011] [InSPIRE].

[28] T. Kobayashi and T. Takahashi, Runnings in the Curvaton, JCAP 06 (2012) 004 [arXiv: 1203.3011] [INSPIRE].

[29] M. Kawasaki, T. Kobayashi and F. Takahashi, Non-Gaussianity from Axionic Curvaton, JCAP 03 (2013) 016 [arXiv:1210.6595] [INSPIRE].

[30] K. Enqvist, T. Meriniemi and S. Nurmi, Generation of the Higgs Condensate and Its Decay after Inflation, JCAP 10 (2013) 057 [arXiv:1306.4511] [INSPIRE].

[31] M. Drewes and J.U. Kang, The Kinematics of Cosmic Reheating, Nucl. Phys. B 875 (2013) 315 [arXiv: 1305.0267] [INSPIRE].

[32] J. Yokoyama, Fate of oscillating scalar fields in the thermal bath and their cosmological implications, Phys. Rev. D 70 (2004) 103511 [hep-ph/0406072] [INSPIRE].

[33] J. Yokoyama, Can oscillating scalar fields decay into particles with a large thermal mass?, Phys. Lett. B 635 (2006) 66 [hep-ph/0510091] [INSPIRE]. 
[34] K. Mukaida, K. Nakayama and M. Takimoto, Fate of $Z_{2}$ Symmetric Scalar Field, JHEP 12 (2013) 053 [arXiv: 1308.4394] [INSPIRE].

[35] A. Chambers, S. Nurmi and A. Rajantie, Non-Gaussianity from resonant curvaton decay, JCAP 01 (2010) 012 [arXiv:0909.4535] [INSPIRE].

[36] K. Enqvist, S. Nurmi and G. Rigopoulos, Parametric Decay of the Curvaton, JCAP 10 (2008) 013 [arXiv:0807.0382] [INSPIRE].

[37] K. Kohri, D.H. Lyth and C.A. Valenzuela-Toledo, Preheating and the non-Gaussianity of the curvature perturbation, JCAP 02 (2010) 023 [Erratum ibid. 1009 (2011) E01] [arXiv:0904.0793] [INSPIRE].

[38] J. Sainio, Curvaton preheating revisited, Phys. Rev. D 85 (2012) 123515 [arXiv:1203.5316] [INSPIRE].

[39] G.W. Anderson and L.J. Hall, The Electroweak phase transition and baryogenesis, Phys. Rev. D 45 (1992) 2685 [INSPIRE].

[40] L. Kofman, A.D. Linde and A.A. Starobinsky, Towards the theory of reheating after inflation, Phys. Rev. D 56 (1997) 3258 [hep-ph/9704452] [INSPIRE].

[41] D.I. Podolsky, G.N. Felder, L. Kofman and M. Peloso, Equation of state and beginning of thermalization after preheating, Phys. Rev. D 73 (2006) 023501 [hep-ph/0507096] [INSPIRE].

[42] D.H. Lyth, Can the curvaton paradigm accommodate a low inflation scale?, Phys. Lett. B 579 (2004) 239 [hep-th/0308110] [INSPIRE].

[43] K. Enqvist, K. Kainulainen and V. Semikoz, Neutrino annihilation in hot plasma, Nucl. Phys. B 374 (1992) 392 [INSPIRE].

[44] K. Dimopoulos, G. Lazarides, D. Lyth and R. Ruiz de Austri, Curvaton dynamics, Phys. Rev. D 68 (2003) 123515 [hep-ph/0308015] [INSPIRE].

[45] R.R. Parwani, Resummation in a hot scalar field theory, Phys. Rev. D 45 (1992) 4695 [Erratum ibid. D 48 (1993) 5965] [hep-ph/9204216] [INSPIRE].

[46] P. Elmfors, K. Enqvist and I. Vilja, Thermalization of the Higgs field at the electroweak phase transition, Nucl. Phys. B 412 (1994) 459 [hep-ph/9307210] [InSPIRE].

[47] A.A. Starobinsky, Multicomponent de Sitter (Inflationary) Stages and the Generation of Perturbations, JETP Lett. 42 (1985) 152 [INSPIRE].

[48] M. Sasaki and E.D. Stewart, A General analytic formula for the spectral index of the density perturbations produced during inflation, Prog. Theor. Phys. 95 (1996) 71 [astro-ph/9507001] [INSPIRE].

[49] M. Sasaki and T. Tanaka, Superhorizon scale dynamics of multiscalar inflation, Prog. Theor. Phys. 99 (1998) 763 [gr-qc/9801017] [INSPIRE].

[50] D.H. Lyth, K.A. Malik and M. Sasaki, A General proof of the conservation of the curvature perturbation, JCAP 05 (2005) 004 [astro-ph/0411220] [INSPIRE].

[51] M. Sasaki, J. Valiviita and D. Wands, Non-Gaussianity of the primordial perturbation in the curvaton model, Phys. Rev. D 74 (2006) 103003 [astro-ph/0607627] [INSPIRE].

[52] For the LHC implications for Higgs-portal dark matter, see e.g. A. Djouadi, O. Lebedev, Y. Mambrini and J. Quevillon, Implications of LHC searches for Higgs-portal dark matter, Phys. Lett. B 709 (2012) 65 [arXiv:1112.3299] [INSPIRE] and references therein. 